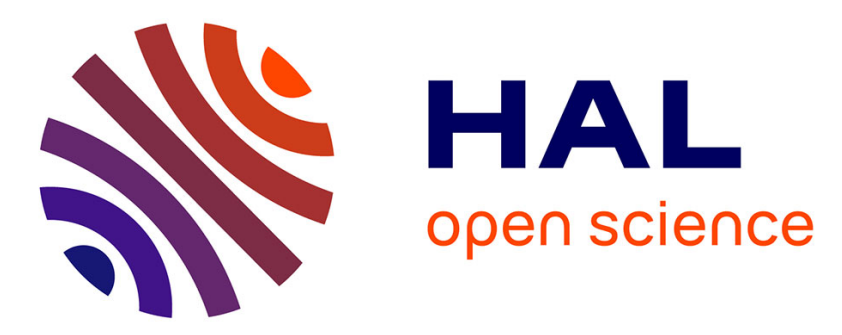

\title{
Synchronization of a thermoacoustic oscillator by an external sound source
}

Guillaume Penelet, Tetsushi Biwa

\section{To cite this version:}

Guillaume Penelet, Tetsushi Biwa. Synchronization of a thermoacoustic oscillator by an external sound source. American Journal of Physics, 2013, 81 (4), pp.290-297. 10.1119/1.4776189 . hal-02057364

\section{HAL Id: hal-02057364 \\ https://hal-univ-lemans.archives-ouvertes.fr/hal-02057364}

Submitted on 5 Mar 2019

HAL is a multi-disciplinary open access archive for the deposit and dissemination of scientific research documents, whether they are published or not. The documents may come from teaching and research institutions in France or abroad, or from public or private research centers.
L'archive ouverte pluridisciplinaire HAL, est destinée au dépôt et à la diffusion de documents scientifiques de niveau recherche, publiés ou non, émanant des établissements d'enseignement et de recherche français ou étrangers, des laboratoires publics ou privés. 


\title{
Synchronization of a thermoacoustic oscillator by an external sound source.
}

\author{
G. Penelet ${ }^{1, *}$ and T. Biwa ${ }^{2}$ \\ ${ }^{1}$ LUNAM Université, Université du Maine, CNRS UMR 6613, \\ Laboratoire d'Acoustique de l'Université du Maine, \\ Avenue Olivier Messiaen, 72085 Le Mans Cedex 9, France \\ ${ }^{2}$ Department of Mechanical Systems and Design, Tohoku University, 980-8579 Sendai, Japan
}

(Dated: November 30, 2012)

\begin{abstract}
Since the pioneering works of Christiaan Huygens about the sympathy of pendulum clocks, synchronization phenomena have been widely observed in nature and science. In this paper, we describe a simple experiment which exhibits several aspects of synchronization when making use of a thermoacoustic oscillator driven by an external sound source. Both the synchronization region of leading order around the natural frequency $f_{0}$ of the oscillator and the ones of higher order (around $f_{0} / 2$ and $\left.f_{0} / 3\right)$ are measured as functions of the loudspeaker voltage and frequency. We also show that increasing the coupling between the loudspeaker and the thermoacoustic oscillator gives rise under some circumstances to the death of self-sustained oscillations (quenching). Moreover, two additional set of experiments are performed : the first one aims at investigating the effect of a feedback loop for which the signal captured by the microphone is delivered to the loudspeaker through a phaseshifter; the second set of experiments aims at investigating the non-trivial interaction between the loudspeaker and the thermoacoustic oscillator when this last one acts as a relaxation oscillator (spontaneous and periodic onset/damping of self-sustained oscillations). The experiment is easy to build and highly demonstrative; it might be of interest for classroom demonstrations or physics lab dealing with nonlinear dynamics.
\end{abstract}

PACS numbers: 43.35.Ud, 05.45.-a

\section{INTRODUCTION}

Synchronization refers to the phenomenon by which a self-sustained oscillator may change its natural frequency when it is coupled to another system oscillating with a different frequency. This process was reported for the first time by Christiaan Huygens ${ }^{1,2}$ who observed that two pendulum clocks hanging from the same beam synchronized mutually: when coupled to each other, the frequency of each pendulum changed slightly in order that they vibrated in perfect harmony, while their motion were opposite (anti-phase locking). Since the pioneering works of Huygens, the process of synchronization has been evidenced or used advantageously in many fields of science, ${ }^{3,4}$ including chemistry (e.g. the BelousovZhabotinsky reaction), physics (lasers), medicine (artificial pacemakers), biology (synchronization of singing crickets), electronics engineering (synchronization of triode generators), or even in social life (clapping audience). The research devoted to this process is currently still active. $^{4,5}$

Synchronization is a well-known but not widely appreciated process which can be described within the common framework of nonlinear dynamics, and it is of interest for teaching needs to have at one's disposal some demonstration devices which are paradigmatic for elementary problems (synchronization of a nonlinear oscillator by an external force, mutual synchronization of oscillators, chaotic synchronization,....). For example, a simple and low-cost experiment exhibiting mutual synchonization of two metronomes resting on a plate that sits on two soda cans has been proposed, ${ }^{7}$ while the famous Van der Pol oscillator excited by an external periodic force ${ }^{8}$ can be nowadays realized with operational amplifiers. ${ }^{9}$

Synchronization processes have also been studied in the field of acoustics: the mutual synchronization of organ pipes has been studied more than a century ago by Lord Rayleigh $^{6}$ (and revisited recently ${ }^{10,11}$ ): he notably reported for the first time the quenching effect (or beating death ${ }^{2}$ ), which refers to the quasi-suppression of oscillations due to the nonlinear interaction of the oscillators. Organ pipes, brass or reed musical instruments are excellent examples of self-sustained acoustic oscillators but another interesting one is the thermoacoustic oscillator, a modern version of the Sondhauss tube. ${ }^{12} \mathrm{~A}$ thermoacoustic oscillator is basically composed of a gas column in a pipe partially filled with a stack of solid plates: the onset of thermoacoustic instability builds up when a critical temperature gradient is applied along the stack, and the frequency of self-sustained acoustic oscillations generally corresponds to the first resonance of the gas column. This device has been extensively studied for the past three decades because it can be used as a new kind of thermodynamic engine, ${ }^{13}$ but it is also interesting as an autonomous oscillator excited by heat. Most of the research works made about thermoacoustic oscillators have dealt with their comprehension and optimization ${ }^{14}$ for energetic use, but one can also find a few papers in which synchronization processes are considered. ${ }^{15-19}$ Spoor and Swift ${ }^{19}$ took advantage of the mutual synchronization of two thermoacoustic engines in order to cancel the vibrations of the pipes induced by high amplitude acoustic waves. Yazaki et al. ${ }^{15,16}$ reported that Taconis oscillations, thermoacoustic oscillations observed at cryogenics temperatures, exhibit synchronization and 


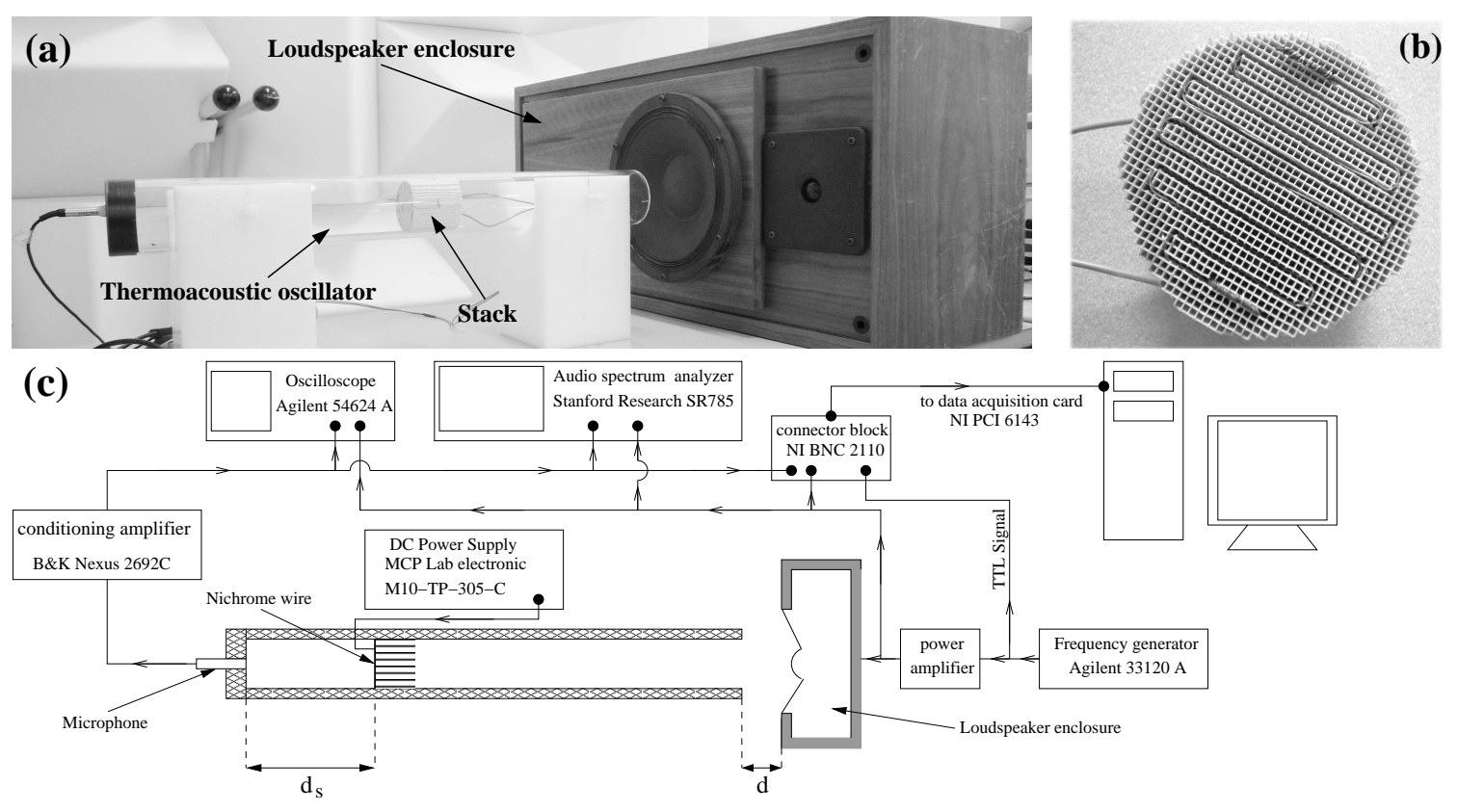

FIG. 1. (a) Photograph of the experimental set-up. (b) photograph of the hot side of the stack. (c) Sketch of the complete experimental set-up.

chaotic dynamics when forced by external oscillations. Müller and Lauterborn ${ }^{17,18}$ made an experimental study of the thermoacoustic oscillator coupled through a small hole to an electrodynamic loudspeaker excited by a sinusoidal voltage with varying amplitude and frequency. Most of the works presented in the present paper are very close, and complementary, to those made by Müller and Lauterborn, but the main motivation here is to present a simple apparatus that would be of interest for demonstration in classrooms. The experimental device is the simplest thermoacoustic oscillator one can build, which only requires a glass tube, a piece of ceramic catalysts and a nichrome wire, while the study of its interaction with an external oscillating force only requires a loudspeaker and basic instrumentation.

In the following, we examine the nonlinear interaction between a loudspeaker and a thermoacoustic oscillator. This interaction is studied as a function of both the driving force (loudspeaker voltage) and the driving frequency, which allows to draw the so-called Arnold Tongues. ${ }^{4}$ The differences in the transition to synchronization for weak forcing (saddle-node bifurcation) or strong forcing (Hopf bifurcation) are highlighted. It is also shown that when the distance between the loudspeaker and the open end of the pipe is short, it is possible to observe the quenching process within a certain range of driving force and frequency. Experiments are also performed using a feedback loop between the microphone and the loudspeaker: the insertion of a phase-shifting circuit along this feedback loop allows the control of the amplitude of selfsustained acoustic waves, ${ }^{20}$ including the possibility of beating death. Finally, a last set of experiments is pre- sented, which aims at investigating the nonlinear interaction between the loudspeaker and the thermoacoustic oscillator when this last one acts as an integrate-and-fire oscillator: ${ }^{4}$ this particular case corresponds to a specific regime for which periodic bursts of (instead of stable) self-sustained acoustic oscillations are generated spontaneously in the resonator. It is shown that the external action of the loudspeaker does not only lead to synchronization but also to the stabilization of acoustic oscillations.

\section{EXPERIMENTS}

\section{A. Experimental apparatus}

The thermoacoustic oscillator considered herein is the so-called acoustic laser, ${ }^{21}$ which is very easy to build with minimum equipment. A photograph of the complete experimental apparatus is given in Fig. 1(a). The system under study consists of a glass tube (length $L=49 \mathrm{~cm}$, inner radius $r_{i}=26 \mathrm{~mm}$, outer radius $r_{e}=30 \mathrm{~mm}$ ) open to free space at one end, and closed by a rigid plug at the other end. The core of the engine, i.e. the stack, is an open cell porous cylinder (radius $r_{i}$, length $x_{s}=48 \mathrm{~mm}$ ) which is inserted into the waveguide. This stack is made up of a 600 CPSI (Cells Per Square Inch) ceramic catalyst with numerous square channels of section $0.9 \mathrm{~mm}$ $\times 0.9 \mathrm{~mm}$. In this device, imposing a large temperature gradient along the stack leads to the onset of selfsustained acoustic waves oscillating at the frequency $f_{0}$ of the most unstable acoustic mode (generally, $f_{0} \approx c_{0} / 4 L$ 
where $c_{0}$ stands for the adiabatic sound speed at room temperature $\left.T_{\infty}\right)$. As illustrated in Fig. 1(b), heat is supplied to the side of the stack facing the plug using an electrical heat resistance wire (nichrome wire, $36 \mathrm{~cm}$ in length, $0.25 \mathrm{~mm}$ in diameter, resistivity $=7 \Omega / \mathrm{ft}$ ) regularly coiled through the stack end, and connected to a DC electrical power supply. Sound is captured using a $1 / 4$ inch condenser microphone (model GRAS type 40BP) flush-mounted through the plugged end of the resonator. Forced synchronization is achieved with a loudspeaker enclosure (Cabasse, type Brick M7) placed at a distance $d$ of the open end of the tube. Data monitoring is realized with an oscilloscope and an audio spectrum analyzer while both the oscillating pressure and loudspeaker voltage are recorded with a data acquisition card, as illustrated in Fig. 1(c).

The post-processing of raw data consists in calculating both the Fast Fourier Transform (FFT) and the Hilbert transform of the sampled data. This enables the computation of the analytic signals

$$
\begin{gathered}
p_{a n a}(t)=p(t)+i p_{H}(t)=A_{p}(t) e^{i \Phi_{p}(t)}, \\
U_{a n a}(t)=U(t)+i U_{H}(t)=A_{u}(t) e^{i \Phi_{u}(t)},
\end{gathered}
$$

of both acoustic pressure $p(t)$ and loudspeaker voltage $U(t)$, where $p_{H}$ and $U_{H}$ stand for the Hilbert transforms of $p$ and $U$, respectively. The relevant parameters which will be used in the following to analyze synchronization processes are the instantaneous phases $\Phi_{p, u}(t)$ and amplitudes $A_{p, u}(t)$.

\section{B. Measurement of the Arnold tongues}

As discussed earlier, the defining feature of synchronization processes is the variation of the natural frequency $f_{0}$ of the autonomous oscillator due to the external action of a periodic force with frequency $f$. This process can occur when $f \approx f_{0}$ (synchronization of the order $1: 1)$ so that the natural frequency $f_{0}$ slips to the frequency $f$ of the external force, but it can also be observed when the forcing frequency is close to a ratio $m / n$ of integer numbers. In other words, the synchronization of order $2: 1$ might be observed when $2 f \approx f_{0}$ so that the frequency of the autonomous oscillator locks to $2 f$. This occurrence of frequency locking depends on both the amplitude of the force and the frequency detuning $f-f_{0}$ (or more generally $n f-m f_{0}$ ). By varying these two parameters, it is then possible to measure the synchronization regions, which are now commonly called the Arnold tongues.

With the present device, it is very easy to change the coupling between the loudspeaker and the thermoacoustic engine by simply varying the distance $d$ [see Fig. 1(c)]. It is also easy to change the position $d_{s}$ of the stack along the resonator, which enables the control of the amplitude and the dynamics of thermoacoustic oscillations. Actually, we observed the higher order synchronizations of
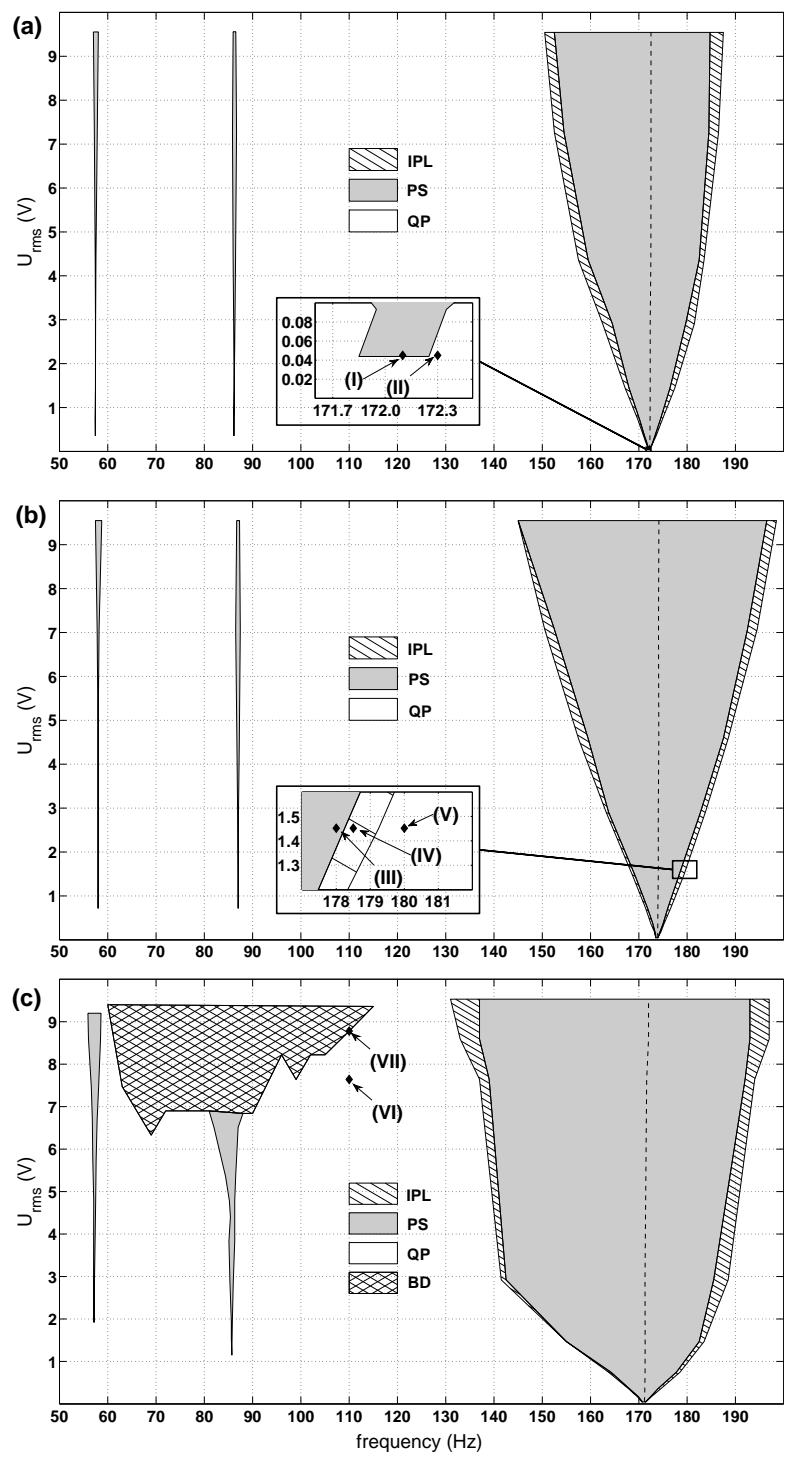

FIG. 2. Arnold tongues obtained in experiments, as a function of the driving frequency $f$ and of the root-mean-square amplitude $U_{r m s}$ of the loudspeaker voltage. The cases (a), (b) and (c) correspond respectively to $\left(d=5 \mathrm{~mm}, d_{s}=8 \mathrm{~cm}\right)$, $\left(d=5 \mathrm{~mm}, d_{s}=19 \mathrm{~cm}\right)$ and $\left(d=1 \mathrm{~mm}, d_{s}=8 \mathrm{~cm}\right)$. The soft grey regions denoted by "PS" correspond to Perfect Synchronization; the regions denoted by "QP" refer to "QuasiPeriodicity" (loss of synchronization); the regions denoted by 'IPL' correspond to "Imperfect Phase Locking" for which the signal looks quasi-periodic but the phase difference stays bounded; the region denoted by "BD" corresponds to "Beating Death" for which the self-sustained oscillations are almost reduced to silence.

$3: 1,2: 1$ and $1: 2$, besides the $1: 1$ synchronization. In Fig. 2, the Arnold tongues obtained in experiments are plotted for three different cases in which both the coupling distance $d$ and the stack position $d_{s}$ are changed.

In order to obtain the above mentioned Arnold tongues, the following experimental procedure was used. 
After having fixed the coupling distance $d$ and the stack position $d_{s}$, all instruments are switched on except for the frequency generator, and the heat power supplied to the nichrome wire is fixed to $Q_{0}=22.6 \mathrm{~W}$ so that stable selfsustained thermoacoustic oscillations are generated in the waveguide at frequency $f_{0}$. After a time delay of about 30 minutes, the frequency generator is switched on and the Arnold tongues are measured by varying the forcing frequency $f$ around $f_{0}$ (or around $n \times f_{0}$ ), and by gradually increasing the loudspeaker voltage. Data acquisitions are performed using a sampling frequency $f_{s}$ which is exactly 30 times the frequency $f$ of the forcing (or $f_{s}=n \times 30 \times f$ in case of higher order Arnold tongues) and the number $N$ of samples is chosen in order that the frequency resolution $f_{s} / N$ be less than $0.1 \mathrm{~Hz}$. It is worth mentioning that the Arnold tongues which are plotted in Fig. 2 do not strictly correspond to the actual Arnold tongues. The first reason is that only a finite number of operating points $\left(f, U_{r m s}\right)$ could be measured and the second reason is that it is impossible to detect the exact bounds of the synchronization regions since the time required to cross the bound with an infinitely small detuning $\delta f$ requires an infinite time. For instance, we observed that for the device initially under a non-synchronous state, and after having changed by $\delta f=0.1 \mathrm{~Hz}$ the forcing frequency, the device could take more than 30 minutes before synchronization was attained. In the experiments, we chose $\delta f=0.1 \mathrm{~Hz}$ as the lower limit of frequency variations, and we did not wait for more than 4 minutes before passing to a new operating point. The results depicted in Fig. 2 have been obtained within a total time of more than 50 hours from a total number of data files which exceeds 1400. Note that an improvement of the experimental protocol could consist in automatizing the experiments over several weeks. ${ }^{11}$

The results depicted in Fig. 2(a) correspond to those obtained when the stack is placed at a distance $d_{s}=8 \mathrm{~cm}$ from the closed end of the resonator, while the distance $d$ between its open end and the loudspeaker is fixed to $5 \mathrm{~mm}$. In the absence of forcing, the frequency of selfsustained oscillations $f_{0}$ equals $172 \pm 0.05 \mathrm{~Hz}$ and the root-mean-square amplitude $p_{\text {rms }}$ of acoustic pressure at the closed end of the resonator equals $292 \pm 5 \mathrm{~Pa}$. Note that after more than 12 hours of measurements in this device configuration, both the frequency and amplitude of acoustic oscillations have slightly drifted to $172.6 \mathrm{~Hz}$ and $330 \mathrm{~Pa}$, respectively, due to a gradual change of the temperature distribution in the thermoacoustic engine (similar variations of $f_{0}$ and $p_{r m s}$ have also been observed in the other experiments). The results depicted in Fig. 2(b) correspond to those obtained with $d_{s}=19 \mathrm{~cm}$ and $d=5 \mathrm{~mm}$ : the initial values of $f_{0}$ and $p_{r m s}$ are 173.5 $\mathrm{Hz}$ and $350 \mathrm{~Pa}$, respectively. From the analysis of the Arnold tongues in Fig. 2(a) and (b), it appears that the position of the stack impacts the width of the leading order Arnold tongue, but the differences between the two set of measurements are not very significant.

Some of the results depicted in Fig. 2(a) and (b) re-
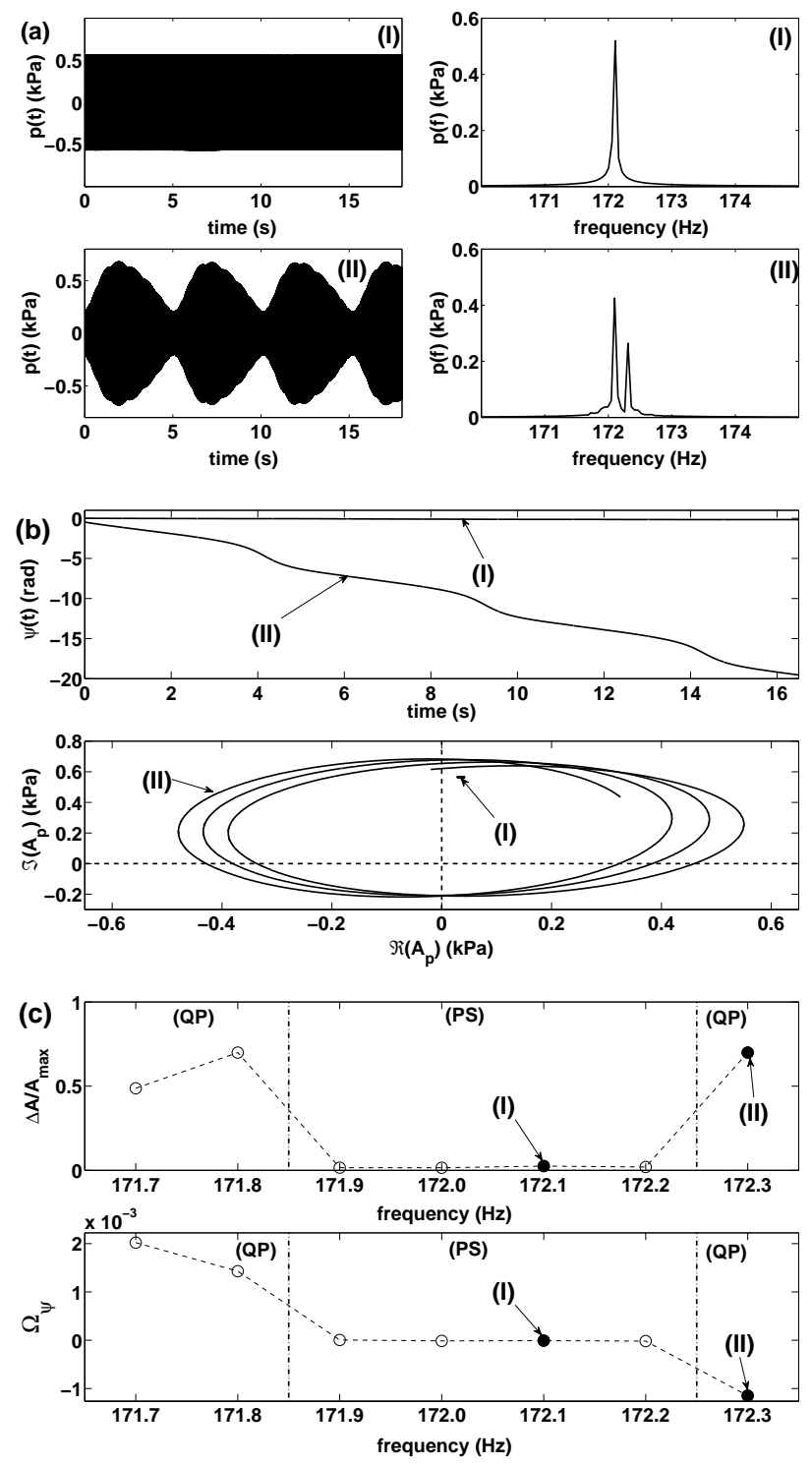

FIG. 3. Transition to synchronization in case of weak forcing $\left(U_{r m s} \approx 40 \mathrm{mV}\right)$. (a) Acoustic pressure $p(t)$ and frequency spectra $p(f)$ measured for the two operating points referred to as $(I)$ and $(I I)$ in Fig. 2(a). (b) Evolution with time of the instantaneous phase difference $\Psi(t)=\Phi_{p}(t)-\Phi_{u}(t)$, and evolution with time of the real part $\Re\left(A_{p}\right)$ of the instantaneous amplitude as a function of its imaginary part $\Im\left(A_{p}\right)$ in the frame rotating at angular frequency $\omega=2 \pi f$. (c) Normalized amplitude modulation $\Delta A / A_{\max }$, and normalized timeaverage phase difference $\Omega_{\Psi}$ as functions of frequency detuning.

quire additional explanations: three different states are drawn in the $\left(f, U_{r m s}\right)$ plane. The first one, denoted as "PS", corresponds to "Perfect Synchronization", for which the instantaneous phase of pressure oscillations locks to that of the oscillating force. The second one, denoted as "QP", corresponds to quasi-periodicity, for which the thermoacousic oscillator keeps its own natu- 
(a)
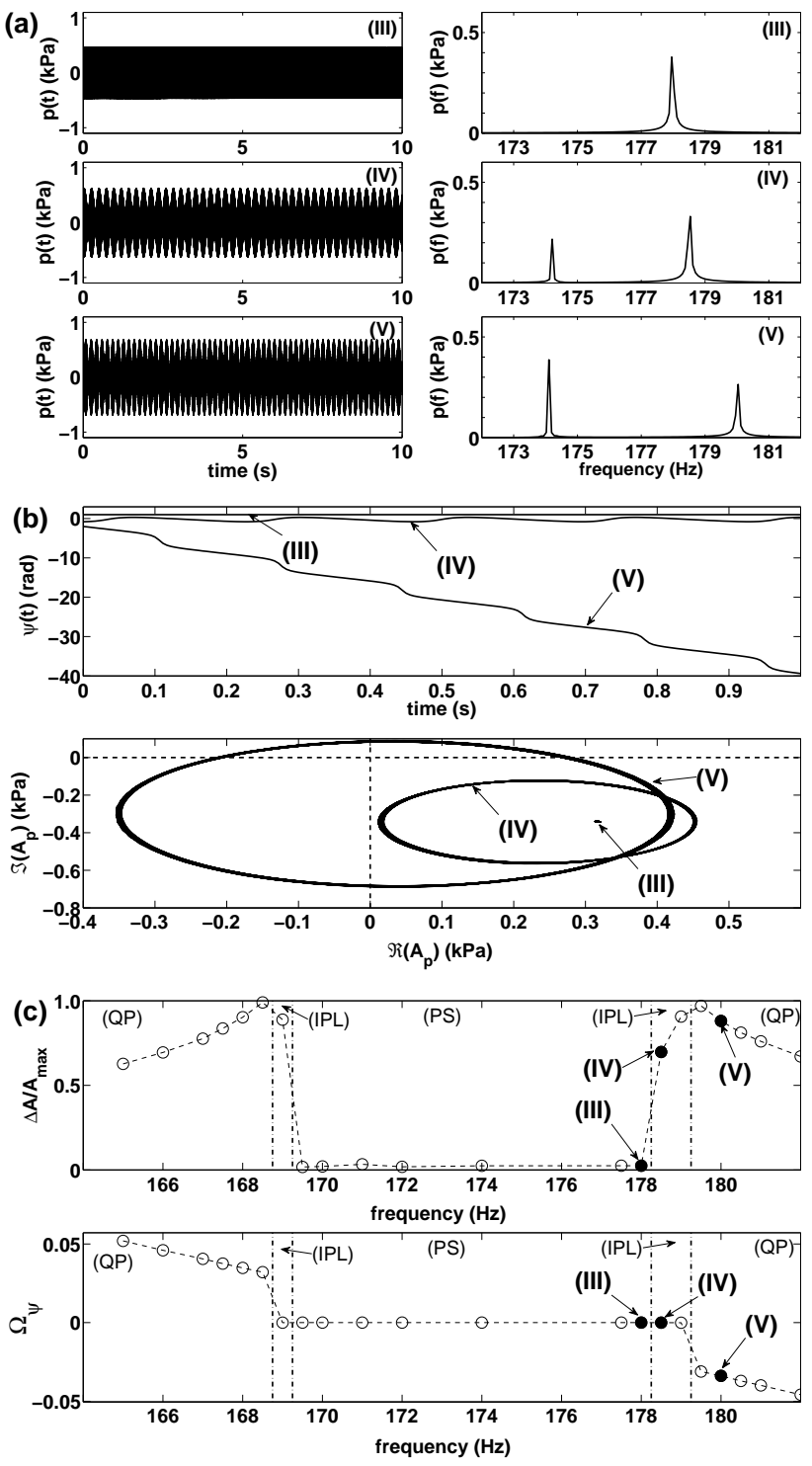

FIG. 4. Transition to synchronization in case of strong forc$\operatorname{ing}\left(U_{r m s} \approx 1.45 \mathrm{~V}\right)$. (a) Acoustic pressure $p(t)$ and frequency spectra $p(f)$ measured for the three operating points referred to as $(I I I),(I V)$ and $(V)$ in Fig. 2(b). (b) Evolution with time of the instantaneous phase difference, and representation in the phase space of the operating points $(I I I)$ to $(V)$. (c) Normalized amplitude modulation, and normalized timeaverage phase difference in function of frequency detuning.

ral frequency $f_{0}$. The last one, denoted as "IPL", corresponds to "Imperfect Phase Locking", for which the instantaneous phase difference $\Psi(t)=\Phi_{p}(t)-\Phi_{u}(t)$ is not constant but stays bounded. These different states are explained with more details in the following.

The transition from perfect synchronization to quasiperiodicity in the case of weak forcing is illustrated by different means in Fig. 3. The most obvious way of analyzing the results consists in looking at the acoustic pressure $p(t)$ together with its FFT amplitude $p(f)$ : this is done in Fig. 3(a) for two operating points referred to as $(I)$ and $(I I)$ in Fig. 2(a). The operating point $(I)$ is within the synchronization region: the pressure signal does not exhibit any amplitude modulation, and the frequency of self-sustained oscillations matches the forcing frequency $f=172.1 \mathrm{~Hz}$. The operating point $(I I)$ is outside the synchronization region: the pressure signal exhibits large amplitude modulations while two distinct peaks are clearly visible on the spectrum. There exists also other ways of analyzing raw data, which are illustrated in Fig. 3(b) for the two operating points $(I)$ and $(I I)$. The first one consists of plotting the instantaneous phase difference $\Psi(t)$ which should be constant in case of perfect synchronization and unbounded in case of quasiperiodicity. ${ }^{4}$ The second one consists in plotting the data in some kind of a phase space, by tracing the real part $\Re\left(A_{p}\right)$ of the instantaneous amplitude of acoustic pressure as a function of its imaginary part $\Im\left(A_{p}\right)$, and in the frame rotating at angular frequency $\omega=2 \pi f$. As illustrated in Fig. 3(b), the phase difference $\Psi(t)$ of the operating point (I) is constant while that of point (II) gradually decreases with time; in the phase space, point (I) is a fixed point while point (II) draws an elliptic limit cycle. Finally, it is also interesting to look at the transition to synchronization as a function of frequency detuning, for a fixed forcing amplitude $U_{r m s}$. In Fig. 3(c), the normalized amplitude modulation $\Delta A / A_{\max }$ and the normalized, time-average phase-difference $\Omega_{\Psi}$ are plotted as a function of the forcing frequency $f$. These two parameters are defined as

$$
\frac{\Delta A}{A_{\max }}=\frac{\max \left(\left|A_{p}(t)\right|\right)-\min \left(\left|A_{p}(t)\right|\right)}{\max \left(\left|A_{p}(t)\right|\right)}
$$

and

$$
\Omega_{\Psi}=\frac{1}{T} \int_{0}^{T}\left(\frac{\Psi(t)}{2 \pi f_{0}}\right) \cdot d t
$$

where the duration $T$ refers to the total duration of data acquisition. The results depicted in Fig. 3(c) show that the loss of synchronization, represented as vertical dotted lines, corresponds to the appearance of beating $(\Delta A \neq 0)$ while the phase becomes unbounded $\left(\Omega_{\Psi} \neq 0\right)$. Note however that frequency increment (or decrement) $\delta f$ is not sufficient here: increasing the number of data points would allow one to check that the evolution of $\Omega_{\Psi}$ with frequency detuning outside synchronization obeys a square-root-law, which is typical of a saddle-node bifurcation expected by theory in the case of weak-forcing. ${ }^{22}$

The transition from perfect synchronization to quasiperiodicity in the case of strong forcing is illustrated in Fig. 4 using the same representations as those of Fig. 3 . The driving voltage is much higher $\left(U_{r m s} \approx 1.45 \mathrm{~V}\right)$, which leads to a more complicated transition. The operating point referred to as $(I V)$ is here particularly interesting. According to Fig. 2(b), this point is within the region called "IPL", but when looking at Fig. 4(a) the motion of the forced nonlinear oscillator looks quasiperiodic. However, it appears from the analysis of $\Psi(t)$ 
in Fig. 4(b) that the instantaneous phase difference $\Psi(t)$ is not constant but bounded, which means that there is an imperfect phase locking. In other words, the nonlinear interaction of both oscillators still corresponds to synchronization, since the frequency of the thermoacoustic oscillator is still controlled by that of the external force. In the phase space diagram, the difference between Imperfect Phase Locking and Quasi-Periodicity is less obvious, since both of the operating points (IV) and (V) correspond to limit cycles, but the the limit cycle of point (IV) does not envelop the origin. ${ }^{22}$ Finally, from the analysis of Fig. 4(c), the transition between perfect synchronization and imperfect phase locking, which operates through a Hopf bifurcation ${ }^{22}$ is clearly visible: it corresponds to the bound for which amplitude modulation appears while the time-average phase difference is still zero.

The results depicted in Fig. 2(c) correspond to those obtained when the stack is placed at a distance $d_{s}=8 \mathrm{~cm}$ from the closed end of the resonator, while the distance $d$ between its open end and the loudspeaker is decreased to $1 \mathrm{~mm}$. In the absence of forcing, we have $f_{0} \approx 171.3 \mathrm{~Hz}$ and $p_{r m s} \approx 230 \mathrm{~Pa}$. The results obtained exhibit significant differences with those of Fig. 2(a) and (b), which means that changing the distance $d$ impacts the nonlinear coupling between the loudspeaker and the thermoacoustic oscillator. First, the Arnold tongues are significantly larger, and the leading order Arnold tongues becomes asymetric (it is not centered around $f_{0}$ for large forcing). Second, a new region appears around the synchronization region $2: 1$, which is called "BD" and corresponds to "Beating Death". The effect of beating death is related to the fact that self-sustained oscillations are almost reduced to silence, and it is illustrated in Fig. 5. In Fig. $5(\mathrm{a})$, the difference between the amplitude of the spectral component at frequency $f_{0} \approx 171.3 \mathrm{~Hz}$ and the one at frequency $f=110 \mathrm{~Hz}$ is plotted as a function of the driving voltage $U_{r m s}$. This difference, $L_{p}(f)-L_{p}\left(f_{0}\right)$, is plotted in terms of Sound Pressure Levels, defined as $L_{p}=20 \log _{10}\left(p / p_{0}\right)$, where $p_{0}=20 \mu \mathrm{Pa}$. From the analysis of Fig. 5(a), we can clearly see that a gradual increase of the driving voltage $U_{r m s}$ leads above some threshold value to the abrupt extinction of self-sustained oscillations which are almost reduced to silence compared to the forced oscillation. When drawing the "BD" zone in Fig. 2(c), we had to choose arbitrarily a threshold value of $L_{p}(f)-L_{p}\left(f_{0}\right)=30 \mathrm{~dB}$ above which it is considered that Beating Death occurs. In Fig. 5(b), the frequency spectra of acoustic pressure are plotted for two operating points referred to as $(V I)$ and $(V I I)$ in Fig. 2(c). Point $(V I)$ is within the quasi-periodicity region and its frequency spectrum show both frequencies $f$ and $f_{0}$ together with their linear combinations. Point $(V I I)$ is within the Beating Death region, and its frequency spectrum shows that the spectral component $f_{0}$ is almost 50 $\mathrm{dB}$ lower than the one at frequency $f$, while all of the combination frequencies have disappeared.

The results depicted in Fig. 2(c) are similar to those
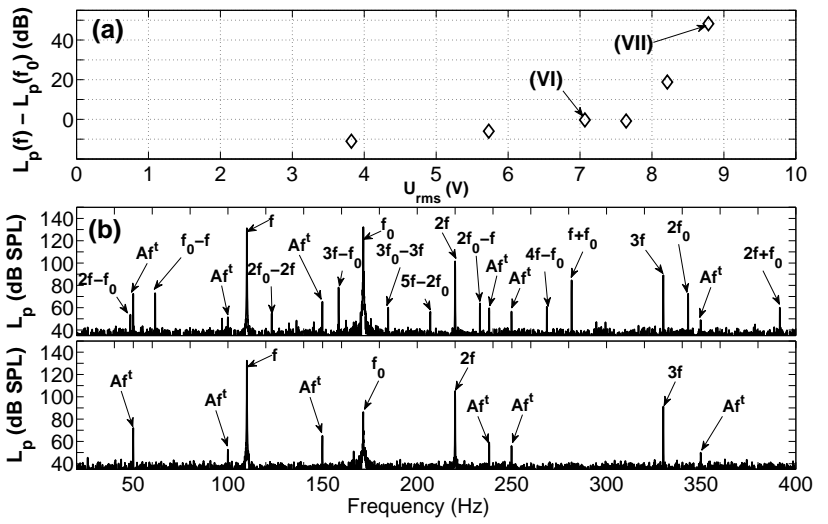

FIG. 5. Illustration of Beating Death in the case of $f=110$ $\mathrm{Hz}$. (a) Measured difference between the sound pressure level $L_{p}(f)$ due to forcing and the sound pressure level $L_{p}\left(f_{0}\right)$ due to self sustained oscillations, as a function of the driving voltage $U_{r m s}$. (b) Frequency spectra corresponding to the operating points referred to as $(V I)$ and $(V I I)$ in Fig. 2(c). Note that the peaks referred to as " $A f^{t}$ " correspond to measurement "artifacts" related to electromagnetic interferences: these peaks are located at the electrical network frequency $(50 \mathrm{~Hz})$ and its harmonics, except for a peak at $f \approx 238 \mathrm{~Hz}$.

obtained by Müller and Lauterborn, ${ }^{17}$ since we observed both $n: 1$ synchronization and beating death in the frequency range around $f_{0} / 2$. However, the shape of the Arnold tongues, or the conditions by which quenching is observed are very different from those obtained in ref. 15: this might be due to the fact that both the thermoacoustic engine and the coupling with the loudspeaker are different in the two studies.

\section{Phenomena in addition to synchronization.}

Apart from the measurements of the Arnold tongues, some additional experiments are interesting to do with this simple thermoacoustic oscillator. In the following, we present two other experiments which aim at investigating additional phenomena other than synchronization.

\section{Feedback loop.}

In Fig. 6, we present the experimental set-up for which the effect of a feedback loop is investigated. In this experiment, as illustrated in Fig. 6(a), the loudpseaker is no longer excited by the frequency generator, but by the signal captured by the microphone itself, and amplified through the audio amplifier. Furthermore, a simple phase-shifting circuit ${ }^{24}$ is inserted along the feedback loop and the effect of the assigned phase shift between the loudspeaker and the microphone on the self-sustained oscillations is investigated. The position of the stack is fixed to $d_{s}=8 \mathrm{~cm}$, the coupling distance $d$ is fixed to 1 
$\mathrm{mm}$, and the heat power $Q_{0}$ supplied to the nichrome wire equals $23 \mathrm{~W}$. Note that this kind of experiment has also been conducted by Müller and Lauterborn, ${ }^{17}$ where the phase shifting circuit was replaced by a multi-effects processor imposing an assigned time delay (instead of a phase shift). However, they did not succeed in initiating self-sustained thermoacoustic oscillations in their experiments.

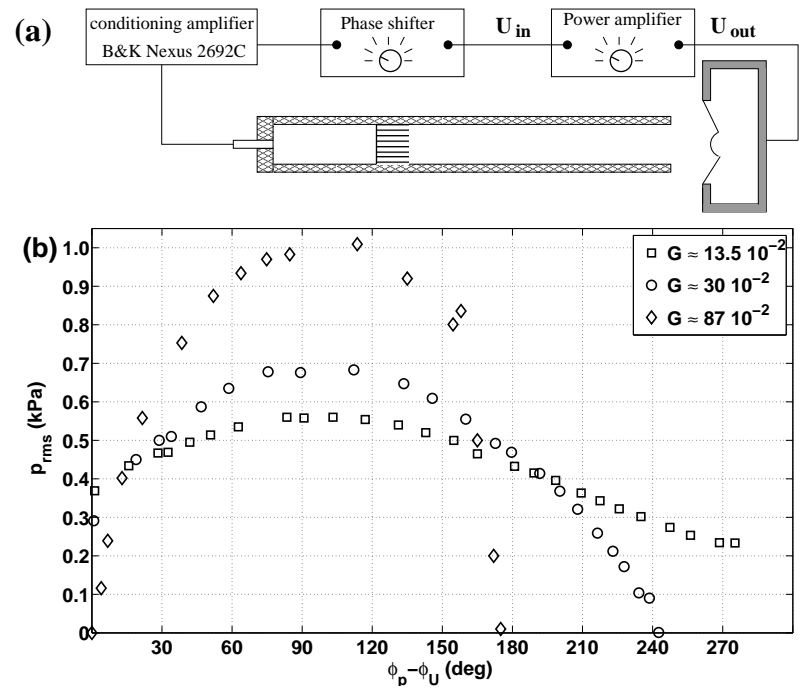

FIG. 6. Effect of a feedback loop. (a) Sketch of the experimental set-up. (b) Steady-state acoustic pressure $p_{r m s}$ as a function of the assigned phase-shift between the loudspeaker and the microphone signals, and for different values of the voltage gain $G=U_{\text {out }} / U_{\text {in }}$, where $U_{\text {in }}$ and $U_{\text {out }}$ refer to the electric voltages at the input and the output of the audio power amplifier, respectively.

In Fig. 6(b), the steady state acoustic pressure is presented as a function of the phase difference between the pressure measured by the microphone and the electrical voltage applied to the loudspeaker. It is presented for different values of the voltage gain $G=U_{\text {out }} / U_{\text {in }}$ [see Fig. 6(a)] monitored by the potentiometer of the audio amplifier. Note that the phase shift cannot be accurately adjusted in our experiments since it is set manually using a potentiometer. Moreover, we could only shift the phase between 0 and $3 \pi / 2$ using this phase shifter. When the voltage gain is set to zero (no feedback loop), the thermoacoustic oscillators sings with a frequency $f_{0} \approx 171.7 \mathrm{~Hz}$. When the voltage gain is set to non-zero values, self-sustained oscillations take place within a certain range of the assigned phase-shift, and the maximum value of the steady state acoustic pressure is reached for $\phi_{p}-\phi_{u} \approx \pi / 2$. This is consistent with our expectations since the acoustic oscillations at the open end of the waveguide should be roughly $\pi / 2$ out of phase with those at the closed end of the waveguide (note also that the loudspeaker itself also induces a small phase shift, which depends on frequency). Moreover, if the voltage gain is increased, the steady state acoustic pressure is increased but the range of phase-shift along which selfsustained oscillations can be observed decreases: for instance, when the voltage gain is fixed to $30 \times 10^{-2}$, selfsustained oscillations are quenched by the loudspeaker as soon as $\phi_{p}-\phi_{u}$ exceeds $240^{\circ}$. We did not make experiments for larger values of $G$, which induced significant distorsion of the signals.

\section{Synchronization in a relaxation regime.}

Another interesting aspect of the thermoacoustic oscillator described in this paper is that it can operate as a relaxation oscillator. When the stack is placed closer to the open end of the resonator and when the heat power supply is fixed to some value slightly above threshold, the amplitude of self-sustained acoustic oscillations are not stable, instead one can observe a spontaneous and periodic onset and damping of thermoacoustic instability. ${ }^{23}$ The physical mechanisms which give rise to this kind of integrate and fire regime are not very clearly understood, but it seems that this effect is due to a competition between the thermoacoustic amplification process induced by heating and the reciprocal effect of acoustic oscillations (thermoacoustic heat transport along the stack, acoustic streaming) which tend to decrease the assigned temperature gradient. In the experiment described below, the effect of external forcing by the loudspeaker is investigated under that particular regime.

The experimental set-up is the same as the one of Fig. 1 (c), but the stack is placed at a distance $d_{s}=25 \mathrm{~cm}$ from the rigid end of the resonator, while the coupling distance $d$ is fixed to $4 \mathrm{~cm}$ and the heat supplied to the nichrome wire is fixed to $Q_{0}=24.5 \mathrm{~W}$. If the loudspeaker is switched off $\left(U_{r m s}=0\right.$, see Fig. 7$)$, an integrate and fire regime of wave amplitude evolution takes place, with a period of about 40 to 50 seconds. The frequency of selfsustained oscillations is around $f_{0} \approx 177.1 \mathrm{~Hz}$, but this frequency actually varies during the process of wave amplification within each burst. For all of the experiments presented in Fig.7, the forcing frequency $f$ is set to 176.9 $\mathrm{Hz}$ (which differs slightly from $f_{0}$ ) and the nonlinear interaction between the loudspeaker and the thermoacoustic oscillator is investigated as a function of the driving voltage $U_{r m s}$. Note that the loudspeaker is systematically switched on at time $t=100 \mathrm{~s}$ while it is switched off (except for the last case) at time $t=150 \mathrm{~s}$. For a very weak forcing $\left(U_{r m s}=4 \mathrm{mV}\right)$, the spontaneous generation of periodic bursts is not disrupted by the forcing. However, self-sustained oscillations do not vanish completely between two bursts, instead a quasi-periodic regime of oscillations takes place. For intermediate amplitudes of loudspeaker voltage $\left(U_{r m s}=10 \mathrm{mV}\right.$ and $U_{r m s}=105$ $\mathrm{mV}$ ), the forcing impacts the integrate and fire regime of wave amplitude evolution: as soon as the loudspeaker is switched on, large amplitude modulations are clearly visible. Moreover, as soon as the loudspeaker is switched off, these modulation are quickly damped, and the inte- 


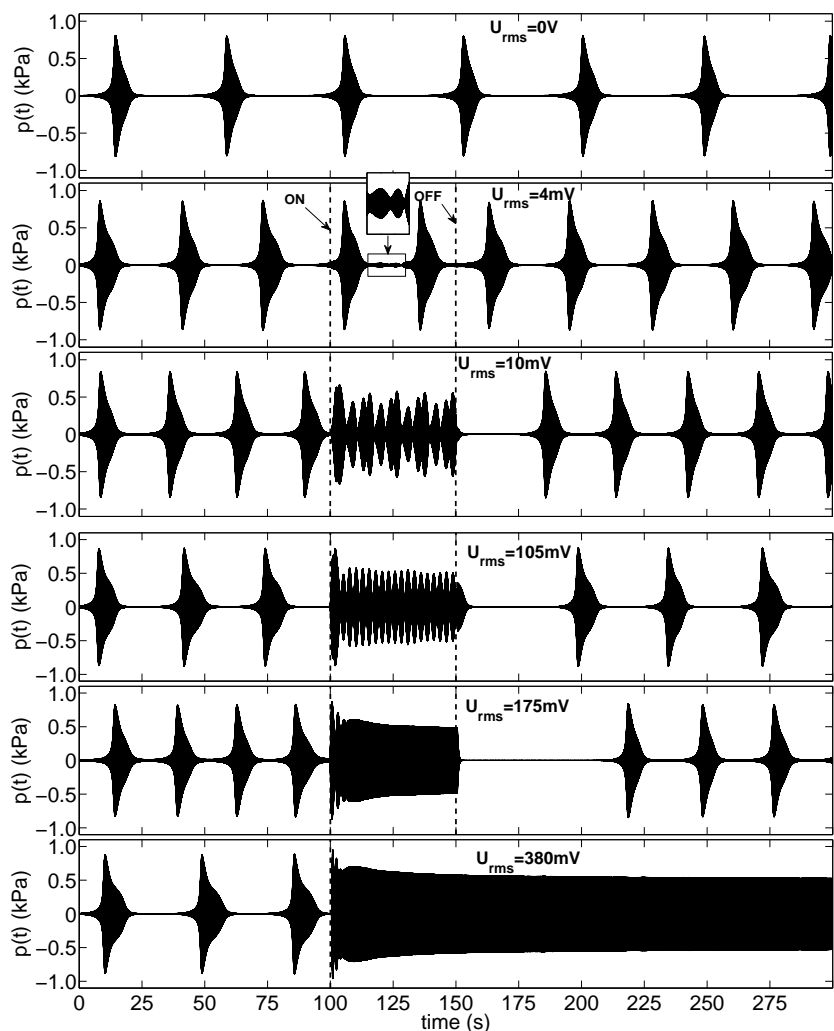

FIG. 7. Synchronization in a relaxation regime. The stack position is fixed to $d_{s}=25 \mathrm{~cm}$ while the coupling distance $d$ equals $4 \mathrm{~cm}$; the heat power supply $Q_{0}$ is fixed to $24.5 \mathrm{~W}$ so that a spontaneous and periodic onset/damping of selfsustained oscillations takes place. From the upper graph to the lower graph, the effect of external forcing (frequency $f=176.9 \mathrm{~Hz}$ ) on the dynamics of wave amplitude evolution is investigated with an increasing louspeaker voltage $U_{r m s}$. Except for the first (no forcing) and the last graph, the loudspeaker is switched on at time $t=100 \mathrm{~s}$, and switched off at time $t=150 \mathrm{~s}$.

grate and fire regime takes place once again. It is however worth noting that some additional time is required before the occurrence of a new regime of periodic bursts. This additional relaxation time increases with the amplitude of forcing, and this is due to the fact that external forcing also disrupts the evolution of the temperature field: heat must be diffused through the device before a new integrate and fire regime is attained. Finally, for larger amplitudes of the loudspeaker voltage $\left(U_{r m s}=175 \mathrm{mV}\right.$ and $U_{r m s}=390 \mathrm{mV}$ ), a new kind of nonlinear interaction is observed. As soon as the loudspeaker is switched on, large modulations of the acoustic pressure are generated, which are however quickly damped: this corresponds to the transition to synchronization, for which the natural frequency of the thermoacoustic oscillator gradually shifts down to the external frequency $f$. Moreover, a less obvious process of wave amplitude stabilization by external forcing is also observed: the spontaneous generation of periodic bursts disappears, instead stable acoustic oscillations occur. Note that if the loudspeaker is switched off $\left(U_{r m s}=175 \mathrm{mV}\right)$, the thermoacoustic oscillator goes back to its integrate and fire regime, but if the external forcing is maintained $\left(U_{r m s}=390 \mathrm{mV}\right)$, then acoustic oscillations stabilize to a finite amplitude. The results depicted in Fig. 7 could be partially interpreted by arguing that when periodic bursts of thermoacoustic oscillations are spontaneously generated, the nonlinear oscillator has two unstable states, i.e. one without oscillations and the other one with finite amplitude oscillations. Due to this, the device switches alternately between the two states, but a weak external force modifies this regime, making one state more stable than the other one.

\section{CONCLUSION.}

We have presented a simple apparatus which exhibits, by sound, several aspects of synchronization phenomena. The results in this paper are not a complete investigation, and additional processes could be explored like, for instance, the effect of a non-sinusoïdal but periodic driving force (or even an aperiodic force ${ }^{25}$ ); a deeper analysis of experimental data would also require to use standard tests in nonlinear dynamics like the calculation of the embedding dimension and of the Liapunov exponents. ${ }^{4,18}$ It is also challenging to find a simple model able to reproduce qualitatively the experimental results obtained above. Finally, it would also be interesting to investigate the mutual synchronization of two thermoacoustic oscillators placed face to face (the adjustment of their frequency could be achieved by replacing the rigid end by a sliding piston).

\section{ACKNOWLEDGMENTS}

The authors would like to thank the scientific council of the Université du Maine, for having attributed a one-month invited professor position to Dr. Biwa. This opportunity allowed us to initiate this study.
* guillaume.penelet@univ-lemans.fr

1 C. Huygens, "Oeuvres Completes de Christiaan Huygens", ed. by M. Nijhoff, Société Hollandaise des Sciences (1893).
2 B. Bennett, M.F. Schatz, H. Rockwood and K. Wisenfeld, "Huygens' clocks", Proc. Roy. Soc. London Ser. A 458, 563-579 (2002). 
${ }^{3}$ I.I. Blekhman, "Synchronization in science and technology", New York, ASME (1988).

4 A. Pikovsky, M. Rosenblum, and J. Kurths, "Synchronization - A Universal Concept in Nonlinear Science", Springer, Berlin (2001).

${ }^{5}$ R.C. Hilborn and N.B. Tufillaro, "Resource Letter: ND-1: Nonlinear dynamics", Am. J. Phys. 65, 822-834 (1997).

6 J.W. Strutt (Lord Rayleigh), "The theory of sound", Dover, New York (1945)

7 J. Pantaleone, "Synchronization of metronomes", Am. J. Phys. 70, 992-1000 (2002).

8 B. Van der Pol and J. Van der Mark, "Frequency demultiplication", Nature 120, 363-364 (1927).

${ }^{9}$ G. Qin, D. Gong, R. Li and X. Wen, "Rich bifurcation behavior of the driven Van der Pol oscillator", Phys. Lett. A 141, 412-416 (1989).

10 M. Abel, S. Bergweiler and R. Gerhard-Multhaupt, "Synchronization of organ pipes: experimental observations and modeling", J. Acoust. Soc. Am. 119, 2467 (2006).

11 M. Abel, K. Ahnert, and S. Bergweiler, "Synchronization of sound sources", Phys. Rev. Lett. 103, 114301 (2009).

12 C.Sondhauss, "Über die Schallschwingungen der luft in erhitzten Glassröhren und in gedeckten Pfeifen von ungleicher Weite", Ann. Phys.(Leipzig) 79, 1 (1850).

13 G.W. Swift, "Thermoacoustics: A unifying perspective for some engines and refrigerators", Acoustical Society of America (2001)

14 S. L. Garrett, "Resource Letter: TA-1: Thermoacoustic engines and refrigerators", Am. J. Phys. 72, 11 (2004).
15 T. Yazaki, S. Sugioka, F. Mizutani, H. Mamada, "Nonlinear dynamics of a forced thermoacoustic oscillation", Phys. Rev. Lett. 64 2515-2518 (1990).

16 T. Yazaki, "Experimental observation of thermoacoustic turbulence and universal properties at the quasiperiodic transition to chaos", Phys. Rev. E 48 1806-1818 (1993).

17 G. Müller and W. Lauterborn, "Experiments with the Thermoacoustic Oscillator Physical and Musical", Proc. Intern. Symp. of Musical Acoustics, Le Normont, Dourdan, France, 178-183 (1995).

18 W. Lauterborn, "Nonlinear dynamics in acoustics", Acta Acust. United Acust. 82, Suppl 1, S46-S55 (1996).

19 P.S. Spoor and G.W. Swift, "The Huygens entrainment phenomenon and thermoacoustic engines", J. Acoust. Soc. Am. 108, 588 (2000).

20 C. Desjouy, G. Penelet and P. Lotton, "Active control of thermoacoustic amplification in an annular engine", J. Appl. Phys. 108, 114904 (2010).

21 S.L. Garrett and R.L. Chen, "Build an Acoustic Laser", Echoes 10(3), 4-5 (2000).

22 A.S. Pikovsky, M.G. Rosenblum and J. Kurths, "Phase synchronization in regular and chaotic systems", Int. Journ. Bifurc. Chaos 10, 2291-2306 (2000).

${ }^{23}$ G. Penelet, M. Guedra, V. Gusev and T. Devaux, "Simplified account of Rayleigh streaming for the description of nonlinear processes leading to steady state sound in thermoacoustic engines", Int. Journ. Heat and Mass Transf. $\mathbf{5 5}, 6042-6053$ (2012).

24 J.S. Lopes, A.A. Melo and V.S. Oliveira, "A simple phase shifting circuit", Phys. Educ. 17, 238-240 (1982).

25 R.V. Jensen, "Synchronization of driven nonlinear oscillators", Am. J. Phys. 70 607-619 (2002). 